\title{
Equipment and Method for Photoelectric Determination of Image Contrast Suitable for Using Square Wave Targets
}

\author{
Fred W. Rosberry
}

(September 1, 1959)

\begin{abstract}
Conventional measurements of the resolving power of lenses employ measuring photographs of test charts containing an array of accurately spaced parallel lines. This method has limited precision because of the variability of photographic emulsions and is timeconsuming in operation. This paper extends previous work by others in obviating these differences by using a direct photoelectric scanning of a line-pattern image formed by the lens under test. Square wave high contrast resolving power targets with two different line pattern arrangements were used as test objects. The image was moved across a stationary slit and photomultiplier tube. The output was recorded as relative transmission.
\end{abstract}

\section{Introduction}

The evaluation of the performance or efficiency of a lens involves, to some degree, the comparison of the output with the input. The respective outputs and inputs are essentially areas of information with input represented by the object or target and the output by the resultant image. A visual comparison of the two represents a typical subjective type of test. An effort was made in the construction of this equipment to minimize the subjective type of test and emphasize the objective. An objective approach includes making some sort of measurements in the object area in order that a numerical comparison might be made with similar measurements in the image. There are several quantities which can be measured in these areas to determine the preservation of information content. It is known that there is a relation between information content and resolving power, the latter of which relates to the smallest distinguishable separation of separate objects and is probably affected by the contrast of the adjacent objects to be distinguished.

Many tvpes of line pattern charts have been developed for testing resolving power. These charts consist of line patterns of varying spacings which proceed in some form of an orderly progression. For example, the ratio of line widths existing between

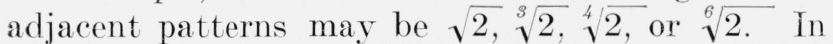
one chart developed at NBS the values of resolving power, indicated by successive line patterns, vary continuously in a linear manner [1]. ${ }^{1}$ Each of these charts has some desirable features. Charts having large steps between successive patterns can cover a longer range of values of resolving power with a relatively small target area as compared to charts of a linear nature or those having small steps between successive patterns. Coarse charts permit a higher degree of repeatability, but a lower degree of precision with respect to the actual limit of resolution [2].

Figures in brackets indicate the literature references at the end of this paper.
The actual determination of the focal location of this resolution limit can be avoided and vet similar results produced by a method which is based on the measurement of contrast in each of the line-space patterns in the image as it approaches the limit. Earlier work along these lines consisted of recording the image on a photographic plate and then scanning or measuring the line-space density with a microdensitometer which recorded the measurements in the form of a curve on graph paper, from which density values could be read [3]. This procedure soon led to the discovery that different types of photographic plates gave different results. The slower, fine-grain emulsions gave higher limits of resolution than did the faster coarse-grain emulsions. Different development procedures applied to the same type of plate would also produce a variation in results. It naturally followed that a procedure to eliminate this photographic variable was necessary. A different type of receiver was needed to supplement the photographic emulsion.

The photoelectric tube had already proven itself a suitable and reliable receiver. The linearity of response for increasing quantities of light is superior in the phototube to that of a photographic emulsion. The work of Schade [4] demonstrated one method in which the photographic process could be eliminated completely: Scanning the image with a narrow slit followed by a phototube measuring the flux which entered the slit. Another modification incorporated in this method is the use of a target which has its ine-space transmission varying along a curve which is essentially sine wave. Targets used previously were square wave in transmission with the dividing edge between line and space a sharp line. The Schade apparatus, on the other hand, uses a rapidly moving target causing a rapid sinusoidal fluctuation in intensity at the receiving slit. The phototube and accessory equipment received and converted this signal, producing on a meter or recorder, a measurement of the amplitude of the 
a-c component. As the test signal emitted from the target is a sine wave and reaches the receiving slit in the same waveform, the effect of the lens under investigation in the system was only to reduce the amplitude of the fluctuations. A comparison of the amplitudes leaving the target with those leaving the lens was handled in a mathematical manner to produce a term called "sine wave response." This equipment is now at the National Bureau of Standards and has been used in a number of investigations. One objection to the system has been the short length of line which it uses. The length of the lines is approximately $2 \mathrm{~mm}$ in the target plane as they are located on the sound track of $35-\mathrm{mm}$ film. Rather than try to make a sine wave target with greater line length, an effort has been made to utilize the square wave target and scan it at a slower speed in order to register each individual line and space. Square wave targets are readily available with line lengths of $8 \mathrm{in}$. and, by using a long target line and relatively short scanning slit, the end effects of the line image are screened out by the scanning slit

In brief, a method has been developed here involving the use of a microphotometer to study the aerial image of long line target transparencies. This method and a few representative results are described in this paper.

\section{Description of Apparatus}

The direct scanning of the image with a phototube was accomplished by arranging the microphotometer as shown in figure 1. A schematic layout is shown in figure 2. The source was a ribbon-filament lamp operated at $6 \mathrm{v}$ and $18 \mathrm{amp}$ by a suitable transformer, with a control for varying lamp intensity. Nine inches in front of the lamp were located the filter holder and condenser system. The target was

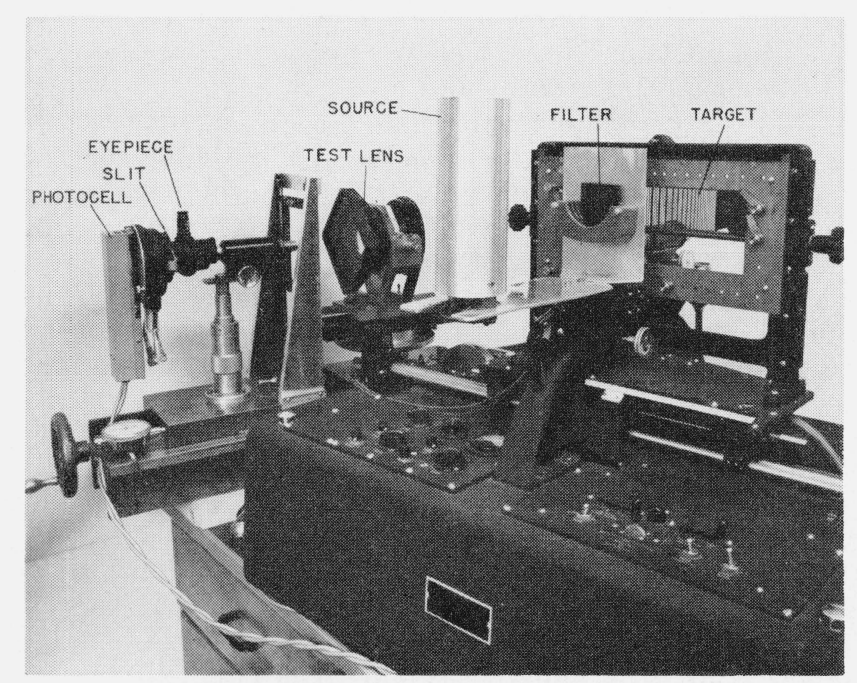

FIGURE 1. Microdensitometer modified to accommodate lamp house and filter holder.

The 3-line target is in place on the instrument cross slide. Test lens and receiving unit can be seen at left.

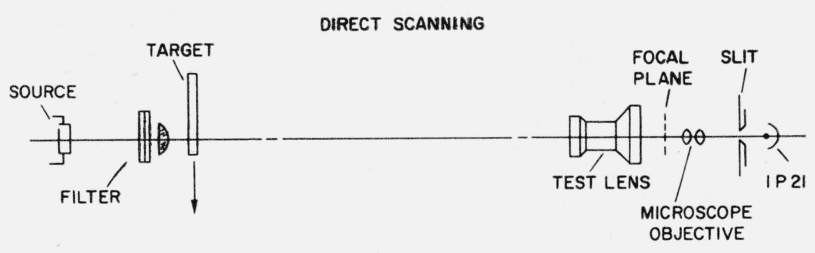

Figure 2. Schematic layout of arrangement of equipment.

The arrow indicates the component in the system which was moved during a test.

mounted on the traveling carriage of the microphotometer and driven by a motor which was synchronized with the paper drive of the recorder. The plane in which the target moved was perpendicular to the optic axis of the test system. The target was so designed that, to cover the required line-space frequency range in the image plane, it was necessary that the target be reduced in size by a factor of 25 , or that it be placed a distance of 26 focal lengths from the lens under investigation. The test lens having a focal length of $6 \mathrm{in}$. thus required a target-to-lens separation of $13 \mathrm{ft}$. Available space in this laboratory did not allow such a spread of equipment, so the system was folded almost back upon itself by the use of a high quality front-surfaced mirror. The next component along the optical axis was the lens under test, which in this investigation was a typical distortion-free wide angle mapping lens including anti-vignetting filter. A microscope was focused on the focal plane of this lens which reimaged the target at $10 \times$ magnification on a slit. Immediately behind the slit, in a light-tight enclosure was a phototube, the output of which was amplified and used as the input to a recorder which traced the transmission of the target image lines and spaces on a chart.

\subsection{Description of Targets}

The two targets used in this work were of the square wave type made at this laboratory. Each is on a 2 - by 10 -in. photographic plate used as a transparency. The geometry of the scanning system requires that only a small portion of the target be illuminated at one time. The only requirement was that the image of the illuminating aperture be larger than the receiving slit and this was relatively easy to accomplish. Another advantage of this system was that the distribution of light flux need not be exactly uniform over the illuminating aperture, as there was no movement of the illuminating aperture with respect to the slit. The targets were both high contrast, with the lines having a density of approximately 3.0 and the clear spaces a density of 0.05 . The target was mounted on the scanning carriage of the microphotometer and moved through the light beam. The target speeds were constant for a given pattern but for coarse patterns the scanning speed was higher than for fine patterns. An effort was made to maintain a constant lines-per-minute scanning rate for the entire target by changing target speed with a gear transmission. 
One of the two targets used was a 2 -in.-wide strip of the multi-line, as shown in figure 3a. The range of this target is from 0.2 to 2.0 lines $/ \mathrm{mm}$ which gave a range, in the image plane of the lens under test, of from 5 to 50 lines $/ \mathrm{mm}$ at 25 times reduction. The second target was a 2 -in.-wide strip of the 75 -line target (fig. 3b) which is in the form of 25 groups of 3 equally spaced lines. The line and space widths were the same in a given group; however, the spatial frequency values varied from one group to the next by a ratio equal to $\sqrt[6]{2}$. The range of resolving power of this target was from 0.2 to 3.2 lines $/ \mathrm{mm}$ and gave a corresponding range in the image plane of 5 to 79.6 lines $/ \mathrm{mm}$.

\subsection{Mirror Assembly}

The front-surface mirror used for folding the optical axis to satisfy space limitations does not show in the schematic diagram or the photographs. It was actually an end mirror borrowed from a 4-in. aperture interferometer. The front surface was of very high quality and flatness, aluminized to enhance the reflecting properties. This mirror was in turn supported on a rigid mount, with provision for minute rotational adjustments about horizontal and vertical axes. Adjustments of this unit were necessary only in the initial alinement of the equipment. The distance from the target to the mirror was roughly the same as the distance of mirror to lens under test, making both legs of the folded system approximately equal. A poor quality mirror at this point in the system would have had a disastrous effect on the results.

\subsection{Lens Assembly}

The lens to be investigated was mounted in a nodal slide as shown in figure 4 and this in turn was mounted on a saddle which slides parallel to the optic axis of the system. When the lens was correctly mounted in the slide, it could be moved about its vertical axis of rotation without displacing the image an appreciable amount to right or left of the receiving slit. This adjustment was not important for data taken only in axial positions as reported in this discussion, but a few trials were made at extraaxial positions. It was found that the equipment functioned equally well in off-axis positions, but results of these extra-axial positions are not reported here.

\subsection{Receiving Unit}

The receiving unit, shown in figure 1, was composed of the microscope, slit, and phototube with associated equipment such as amplifier and recorder. The microscope was of standard design, with the incorporation of an eyepiece at right angles to the line of sight and focused on the slit by means of a mirror, providing an opportunity to examine the image as it was focused on the slit. This feature was also necessary for making adjustments to render the line pattern in the image and the slit parallel. Adjustments could be made by rotating the slit with respect to the image. The microscope objective used in this work was a 10-power, 16-mm apochromat. The slit size was $35-\mu$ wide by $10-\mathrm{mm}$ long, which was somewhat shorter than the image of the

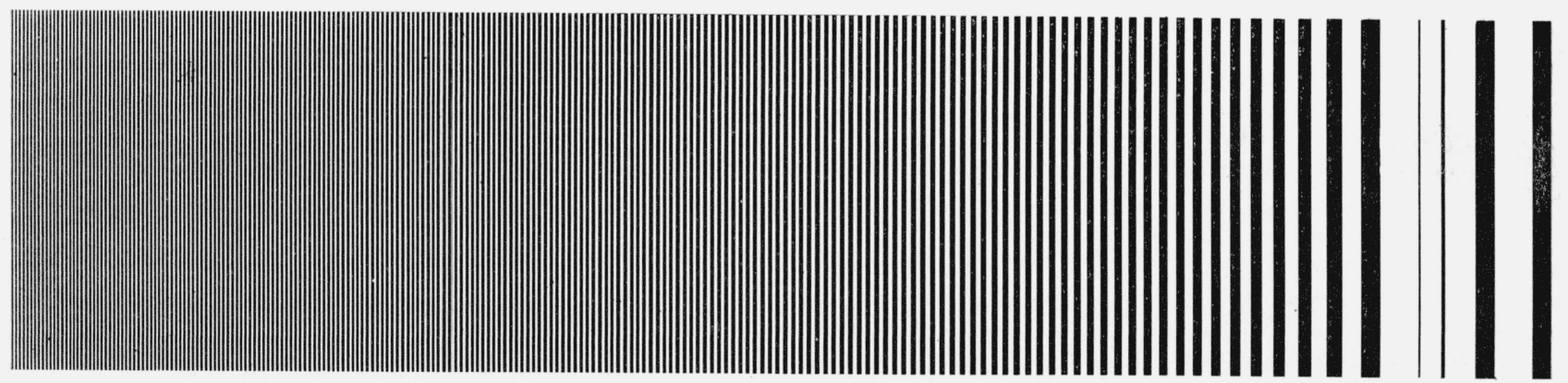

a

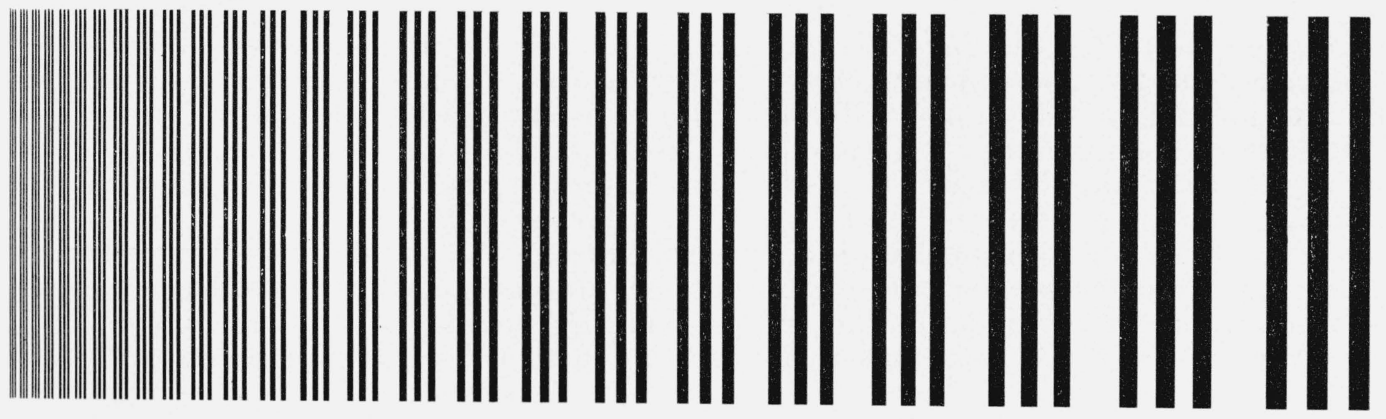

Figure 3. Multiline (a) and 3-line (b) targets used in this investigation. 


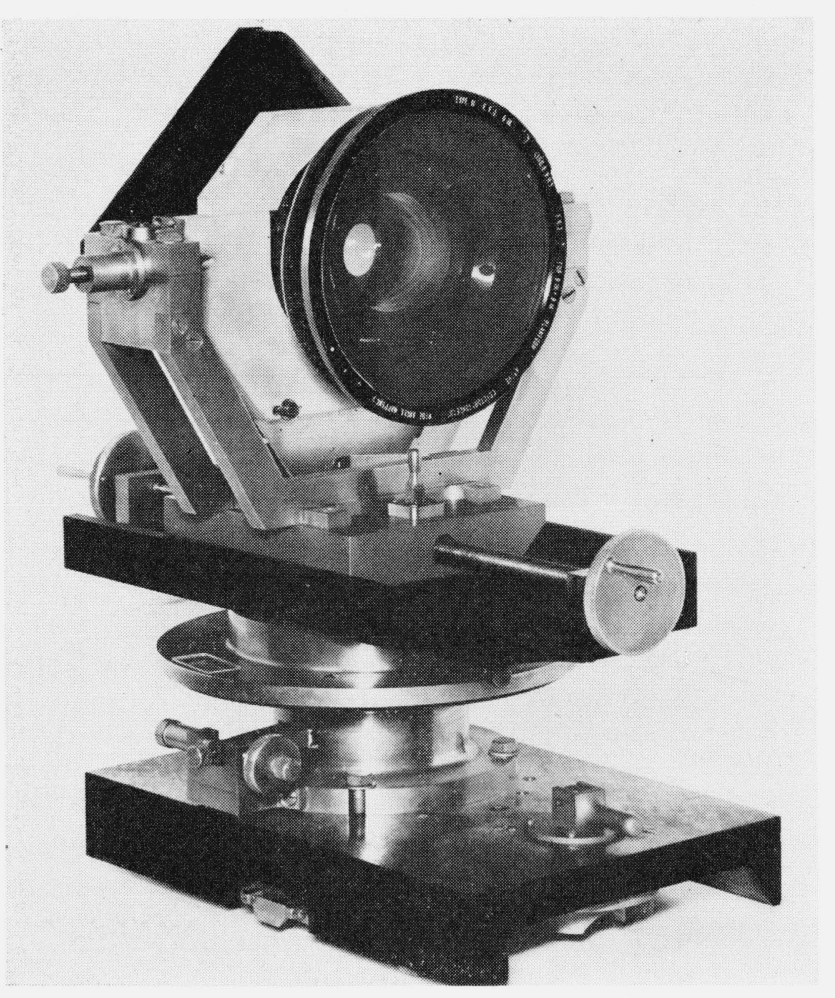

Figure 4. Lens mounted in nodal slide.

target lines, thereby virtually eliminating any of the end effects in the line patterns.

The slit support, phototube enclosure, and microscope were the standard receiving unit on the microphotometer. The phototube enclosure was originally made to accommodate a 921 type of phototube, but this did not have the necessary sensitivity for successfully recording the small flux densities which sometimes passed the somewhat narrow slit. A 1P21 photomultiplier tube was found to give much better results but would not fit into the standard housing. A new rear cover plate was made for the standard housing and the photomultiplier probe unit was attached to this cover. This separated the photomultiplier and slit about 2 in. which was more than a good design should allow. This was corrected by building a tunnel to collect all the flux emerging from the slit and pipe it to the tube. A high reflective coating on the interior of the tunnel reduced losses from scattering to a minimum. The photomultiplier unit had its own amplifier with four different ranges of sensitivity and a control for adjusting the dark current of the phototube. A small adapting unit was all that was necessary to run the microphotometer recorder from this amplifier. The recorder strip chart was a 100-division, uniformly spaced chart, which made the response plotted on a percent transmission scale.

The receiving unit, consisting of microscope, slit, and phototube, was mounted on one saddle similar to the one holding the nodal slide. Movement of one saddle with respect to the other was used as the means of locating a good focal plane of the lens. The nodal slide saddle was locked in position after the initial setup, and all focusing was done with the receiver unit. A dial indicator was fixed to the ways with its indicating probe in contact with the saddle for measuring the small displacements in focal position.

\subsection{Alinement}

Proper alinement of some of the components in this system was very important, while small misalinements in others did not appreciably affect the reliability of the data. The nodal slide and receiving: unit were mounted on a short length of ways. It was important that the axis of these ways were parallel with the optical axis established by the light source and mirror. The target was mounted on the plate carriage of the microphotometer, thus establishing the direction of travel of the plate carriage perpendicular to the optical axis. Perpendicularity was checked by substituting a mirror for the target and adjusting the direction of the ways until reflected light from the source fell back upon itself.

The lens was mounted in the nodal slide with the aid of an axis finder to assure that the optical axis of the lens was parallel with the line of travel of the saddle on the ways. The lens was also located with its nodal point over the center of rotation of the nodal slide, thus assuring that the image would not move laterally from the receiving slit when the lens was rotated to simulate off-axis positions.

The microscope and slit combination were next alined along the axis established by the lens in the nodal slide. Alinement of these two components was important, as they were mounted on two different saddles, and focusing of the test lens was accomplished by varying the distance separating them. Misalinement here resulted in a lateral shift of the image when the lens-to-receiver distance was changed. The components on the ways were now in alinement with each other and it was relatively easy to make this optical axis coincident with the one for the whole system by moving the ways and saddles as a unit.

\subsection{Calibration}

The linearity of the amplifier, phototube, and the recorder was checked as a unit by substituting an adjustable slit for the fixed slit in front of the phototube unit. The space between slit and phototube was suitably masked to exclude all extraneous light, thus allowing only the slit to pass the light registering on the phototube. The recorder was then zeroed for dark current conditions. The slit was opened by small but uniform increments to its maximum opening. The amplifier and recorder thus produced a trace which was read and plotted against slit area to indicate the response of the system. The curve produced by these data verified the expected linearity of the system. 


\section{Description of a Typical Run}

A typical run consisted of determining the response of 5 known spatial frequencies at 11 different focal positions on axis of a common type photographic objective. The response in the image was expressed in terms of contrast between lines and spaces. The target, figure $3 \mathrm{a}$, provided a choice of 200 different spatial frequencies but to shorten the scanning and data handling time only 5 preselected frequencies were accurately measured, skipping over all intervening patterns. During a run the target was the only component in the system which moved. It could be moved slowly during measurement of each of the 5 frequencies and then moved rapidly through those between. The 5 spatial frequencies selected for measurement were $10,20.5,30.5,40$, and 63.5 lines $/ \mathrm{mm}$, but any or all others could have been used. A visual determination was made of the position of best focus and the first scan of the 5 frequencies was made at a focal position $1 \mathrm{~mm}$ back from this position. Starting at this long focal position a scan of the 5 frequencies was made at steps of $0.2 \mathrm{~mm}$, to the other extreme of $1.0 \mathrm{~mm}$ inside of focus. This procedure produced a scan at 11 different focal positions. Measurement of the changes in focal position was accomplished with the dial indicator attached to the ways and bearing against the receiving unit saddle.

The traces produced by the recorder at each focal position were treated the same in that the maximum and minimum light transmission of each line and space of the predetermined frequency patterns was recorded. The contrast of each frequency was determined from the transmission data by using the formula

$$
C=\frac{T_{s}-T_{1}}{T_{s}}
$$

where $T_{s}$ is the transmission of a space and $T_{1}$ of the adjoining line. These contrast data are plotted against spatial frequency in figure 5, which includes the 10 most informative of the 11 focal positions. There is a curve for each focal position, showing the manner in which the contrast decays toward zero. The larger numbers along the ordinate axis indicate the focal position and the smaller positive and negative numbers indicate the contrast scale. The abscissae represent the frequency in lines per millimeter. It will be noted that portions of the curves drop below the zero contrast value and have negative values. The negative values indicate the areas in which spurious resolution [5] is present and are only on the extreme short focus positions for this particular lens.

This equipment was found to lend itself very well to producing evidence showing what is taking place in these areas of spurious resolution. Two microphotometer traces of two aerial images are shown in figure 6 . The trace which has the larger amplitudes represents a focal position close to best focus,

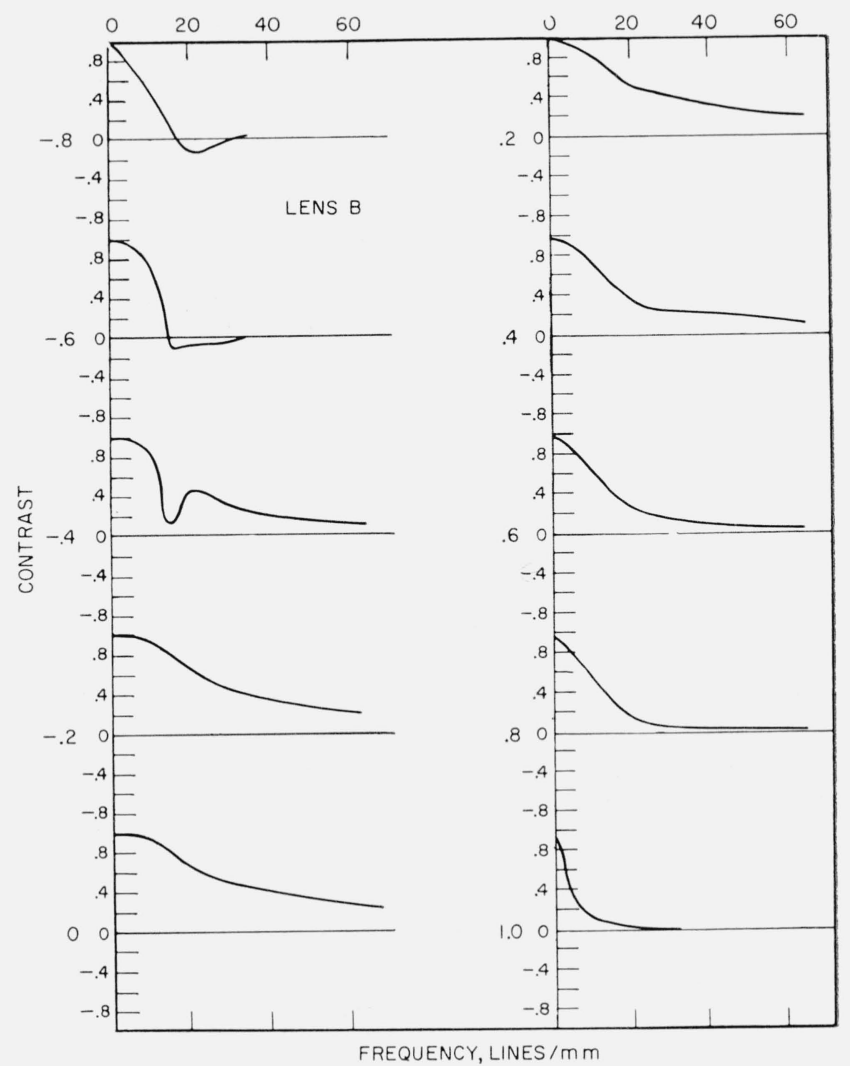

Figure 5. A set of curves showing the contrast at various spatial frequencies of the direct scanned image as recorded at 10 different focal positions.

Large numbers on the ordinate represent focal positions in millimeters.

and forms a good representation of the actual target in use as each line and space is registered in its relative position. The inner or low-amplitude trade represents a focal position $1.2-\mathrm{mm}$ closer to the lens than the other trace. The two traces have been put in proper register with the maximum of the first line for the two traces in coincidence. It can be seen by following the inner trace that, for parts of the range of spatial frequency, the lines and spaces are registered out of phase and a line is indicated where a space should be. This indicates that, in this first zero point or node, one-half line has been lost, and the indicated resolution in this area to the next node is $180^{\circ}$ out of phase. This is spurious resolution. At node two, another one-half cycle is lost. The area extending between nodes two and three shows the lines and spaces back in proper register but $360^{\circ}$ out of phase, as one whole cycle has been lost.

The curves in figure 5 show the contrast as negative when the line-space register is out and positive when it is in. All of the contrast curve to the right of the first zero point represents spurious resolution. 


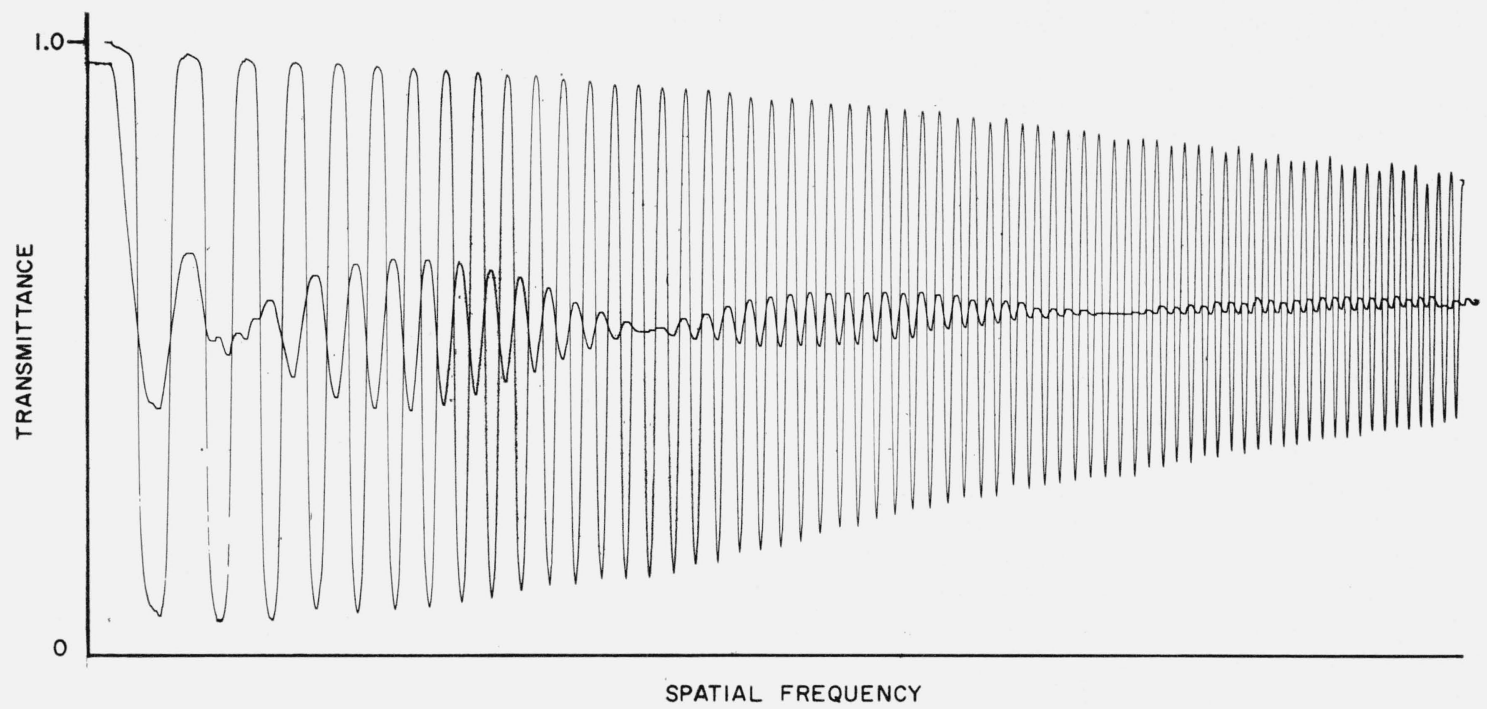

Figure 6. Two microdensitometer traces made by direct scanning of the image.

The trace with greatest magnitude was made at a position near best focus. Inside trace was made at a focal position 1.2-mm nearer lens. Note changes in phase.

\section{Other Uses of Equipment}

It is possible to insert color filters in the light path between the light source and target. The effective area of the receiving slit when projected back to the target is small enough to allow the use of standard 2-in. square filters. With the use of a suitable filter, investigative work can be carried out in a narrow spectral region. The use of several different narrow band filters permits the whole range of white light to be broken into as many narrow bands, which greatly reduces the effects of chromatic aberration within each band.

If repeated measurements of the same frequency pattern are made at different focal positions using the three-line target, it can be assumed that a bestfocus position could be established by locating the position of maximum contrast rendition. This procedure was followed, using a filter and recording traces at 14 established focal positions. It was noted that at the various focal positions the contrast started at a low value, increased to a maximum, and then diminished. The filter was then changed to one which transmitted light in an adjacent spectral region. Another set of traces was taken using the same focal settings and noting the resultant contrast. This procedure was repeated using 6 different spectral regions to cover the range of visible wavelengths. When the 6 traces were arranged on a common focal position scale the results appeared as shown in figure 7. The numbers along the ordinate represent the Wratten filter number used to produce that trace, otherwise the ordinate scale is in transmittance. The spectral range passed by these filter numbers were: Blue with 45 ; green with 16 and 60 ; yellow with 90 ; orange with 25 ; and red with 29 . Further investigation with different $\mathrm{f} /$ values and at off-axis positions could have easily been performed but exploration of the versatility of the equipment was the prime interest at the time.

Another image phenomenon which shows itself rather clearly can be seen by referring to figure 8 . This illustration consists of two runs through focus in which the same spatial frequency pattern was scanned for each run. The upper run was the result of repetitive scans of a relatively coarse pattern of 5 lines $/ \mathrm{mm}$ in the image while the lower run represents the response to the 64 lines $/ \mathrm{mm}$ pattern. The unrestricted spectral range of a tungsten-lamp source was used for these traces. Close examination of the focal location of the two areas of maximum contrast shows a separation of about $0.2 \mathrm{~mm}$. The values of contrast at each focal position could have been determined for these traces and curves of contrast versus focal position drawn to illustrate the same effect. The added work is not necessary here as the raw data alone indicate the results in a satisfactory manner.

The next experiment showing the possibilities of this apparatus involved making two apertures to be used as stops in the lens under investigation. These were constructed by depositicg opaque material in the shape of a circle on thin cover glasses. The diameter of the full aperture of the lens at $f / 6.3$ was measured and an annular ring was deposited which reduced the clear aperture of one-half the original area, or an $\mathrm{f} / \mathrm{value}$ of 8.9 . Another cover glass was used to make a negative of this aperture, which consisted of a circular opaque center with a transparent annular ring to transmit the light. The two apertures contain the same amount of clear area and are therefore the same $\mathrm{f} /$ value.

A full aperture (f/6.3) trace was made by again making repetitive scans of a constant spatial frequency 3 -line pattern at 10 different focal settings 


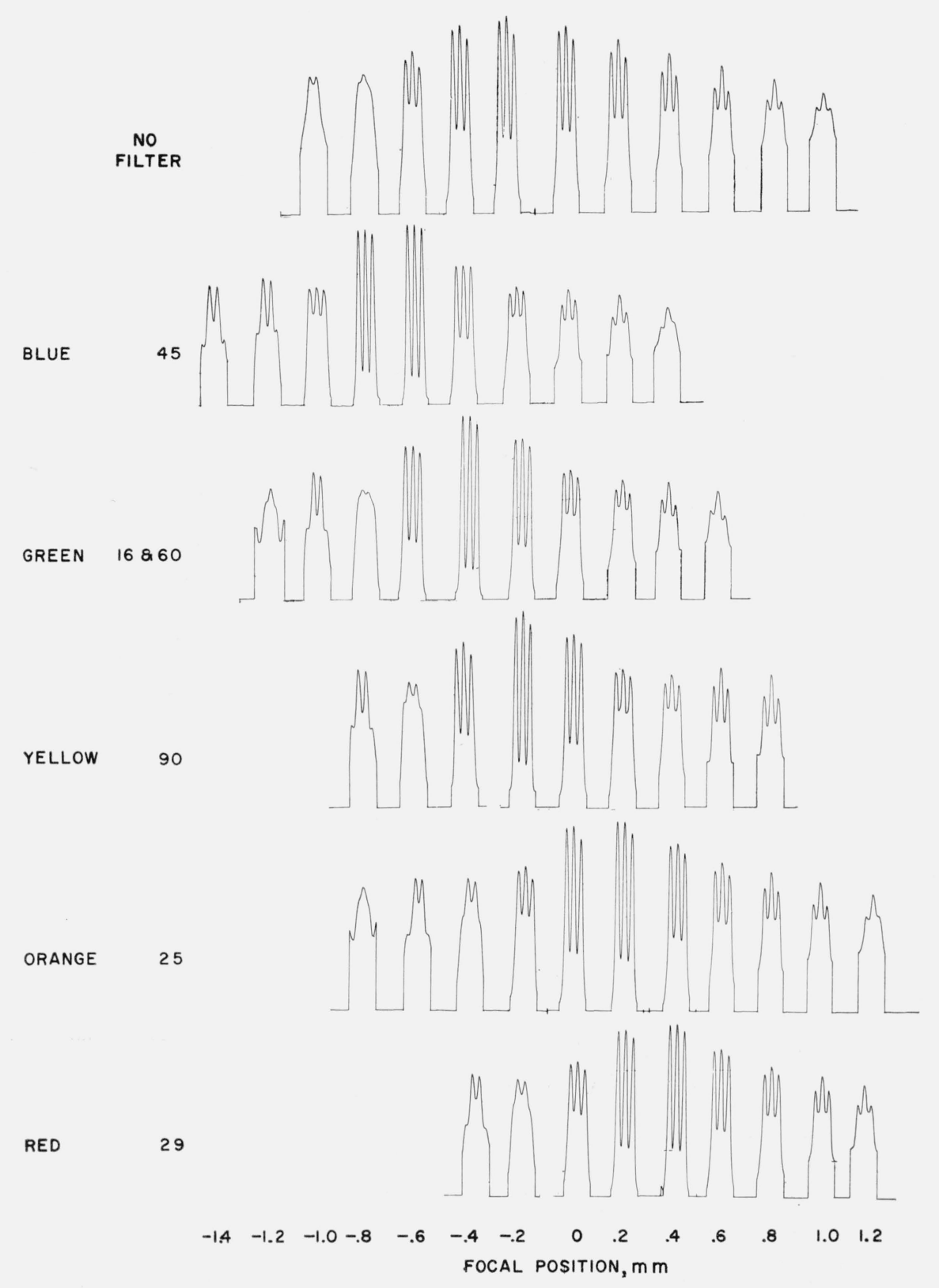

Figure 7. Traces showing the response of a single 3-line pattern made at various focal positions. Each horizontal row was made in a different spectral region indicated by the filter number at the left. Note the shift in position of best focus.

while using a tungsten source as an illuminant. The full aperture trace was made with a clear cover glass in the same position the partially opaqued aperture stops were to occupy in the succeeding two runs. This was done to render constant the effect of the 0.1-mm glass thickness which was added to the lens system. Traces were then made with the two f/8.9 apertures, and the three traces arranged according to a common focal-position scale for comparison as seen in figure 9 . A test along these lines might be used in a study of longitudinal spherical aberration in a lens system.

There are undoubtedly other areas of image evaluation which could be investigated with this equipment. One such area of study is that of star images, which was accomplished by substituting a camera for the photocell. One of the initial aims in building this apparatus was to reduce the time required for a lens test over the photographic method and this has attained that goal. 


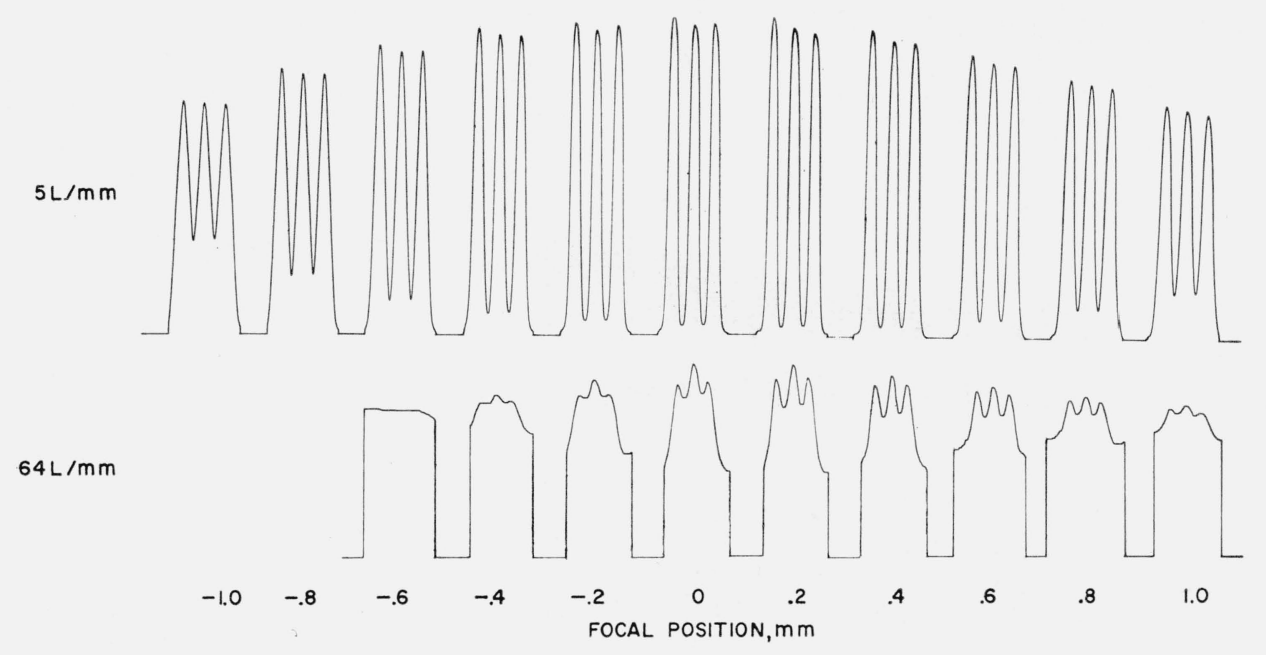

FiguRE 8. Traces showing the response of two frequency patterns as indicated at left.

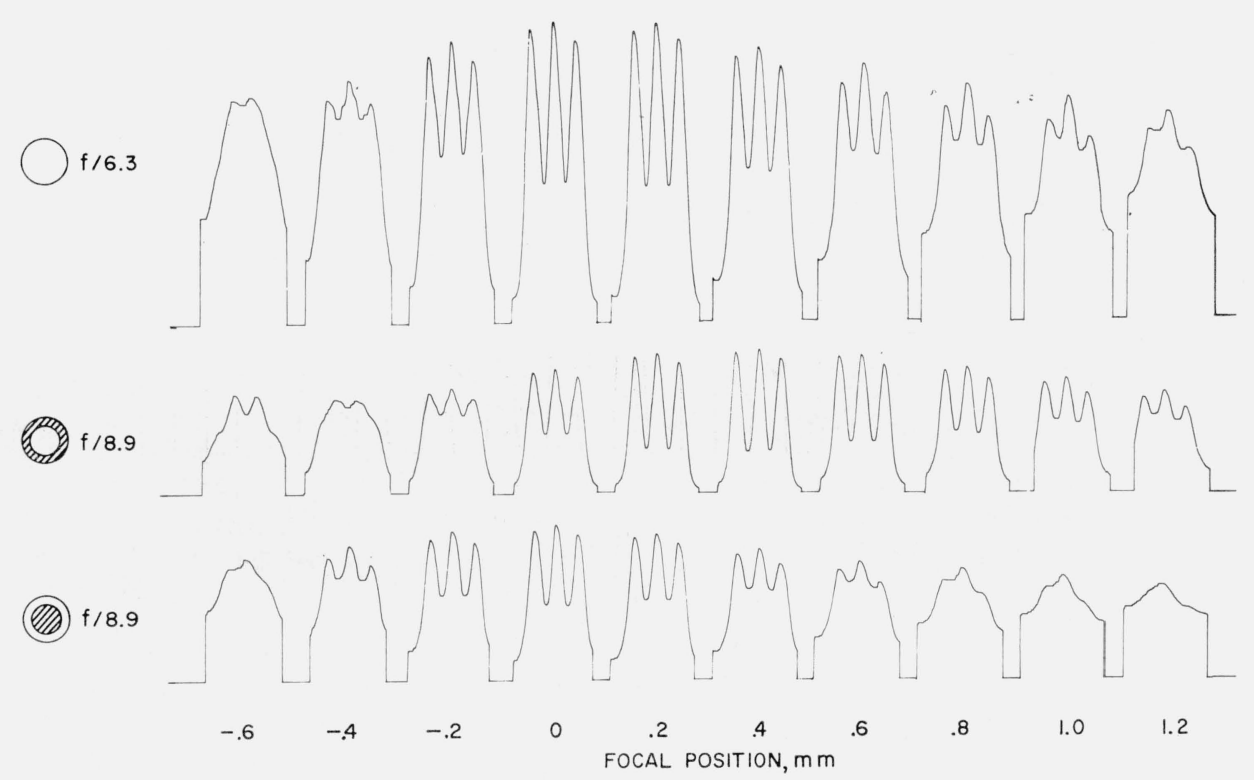

FIGURE 9. Traces showing the response of repeated measurement of a single 3-line pattern at 10 different focal positions.

Each horizontal row was made with a different aperture stop, the first stop was the normal, in the second the aperture was stopped down to $\mathrm{f} / 8.9$, and in the third the stop opening was shaped in the form of an annular ring with the same $\mathrm{f} / \mathrm{value}$ as the second.

\section{References}

[1] F. E. Washer and F. W. Rosberry, New resolving power test chart, J. Opt. Soc. Am. 419 (1951).

[2] F. E. Washer and I. C. Gardner, Method for determining the resolving power of photographic lenses, NBS Circ. 533 (1953).

[3] F. W. Rosberry, Effect of object frequency on focal position of four photographic objectives, J. Research NBS 57 17 (1956) RP2688.
[4] O. H. Schade, A new system of measuring and specifying image definition, NBS Circ. 526 (1954).

[5] R. N. Hotchkiss, F. E. Washer, and F. W. Rosberry, Spurious resolution of photographic lenses, J. Opt. Soc. Am. 419 (1951).

Washington, D.C.

(Paper 64C1-25) 